\title{
The Zhou's Method for Solving the White-Dwarfs Equation
}

\author{
Pedro Pablo Cárdenas Alzate ${ }^{1}{ }^{*}$, William Ardila Urueña ${ }^{2}$ \\ ${ }^{1}$ Department of Mathematics, Universidad Tecnológica de Pereira, Pereira, Colombia \\ ${ }^{2}$ Department of Physics, Universidad Tecnológica de Pereira, Pereira, Colombia \\ Email: *ppablo@utp.edu.co,williamar@utp.edu.co
}

Received March 8, 2013; revised April 8, 2013; accepted April 15, 2013

Copyright (c) 2013 Pedro Pablo Cárdenas Alzate, William Ardila Urueña. This is an open access article distributed under the Creative Commons Attribution License, which permits unrestricted use, distribution, and reproduction in any medium, provided the original work is properly cited.

\section{ABSTRACT}

In this work we apply the differential transformation method (Zhou's method) or DTM for solving white-dwarfs equation which Chandrasekhar [1] introduced in his study of the gravitational potential of these degenerate (white-dwarf) stars. DTM may be considered as alternative and efficient for finding the approximate solutions of the initial values problems. We prove superiority of this method by applying them on the some Lane-Emden type equation, in this case $y^{\prime \prime}+\frac{2}{x} y^{\prime}+\left(y^{2}-C\right)^{3 / 2}=0$. The power series solution of the reduced equation transforms into an approximate implicit solution of the original equation.

Keywords: Zhou’s Method; DTM; Lane-Emden Type Equation; White-Dwarfs

\section{Introduction}

Other classical nonlinear equation which has been the object of much study is called the white-dwarf equation. This equation has the form:

$$
y^{\prime \prime}+\frac{2}{x} y^{\prime}+\left(y^{2}-C\right)^{\frac{3}{2}}=0
$$

with $x>0$ and subject to initial conditions:

$$
y(0)=1, \quad y^{\prime}(0)=0
$$

The Equation (1) is one of Lane-Emden types, where $f(y)=\left(y^{2}-C\right)^{\frac{3}{2}}$. In fact, it reduces to Lane-Emden equation with index $n=3$ when $C=0$.

Let us consider a spherical cloud of gas (see Figure 1) and denote its hydrostatic pressure at a distance $r_{1}$ from the centre by $P$. Let $M\left(r_{1}\right)$ be the mass of the spheres of radius $r_{1}, \phi$ the gravitational potential of the gas, and $\boldsymbol{g}$ the acceleration of gravity.

Then, we have the following equations:

$$
g=\frac{G M\left(r_{1}\right)}{r_{1}^{2}}=-\phi^{\prime}\left(r_{1}\right)
$$

*Corresponding author. where $\boldsymbol{G}$ is the gravitational constant.

Thus, three conditions are assumed for the determination of $\phi$ and $\boldsymbol{P}$ :

$$
\mathrm{d} P=-g \rho \mathrm{d} r_{1}=\rho \mathrm{d} \phi
$$

where $\rho$ is the density of the gas.

$$
\nabla^{2} \phi=\phi^{\prime \prime}\left(r_{1}\right)+\frac{2}{r_{1}} \phi^{\prime}\left(r_{1}\right)=-4 \pi G_{\rho}
$$

and

$$
P=K \rho^{\gamma}
$$

where $\gamma$ and $K$ are arbitrary constants.

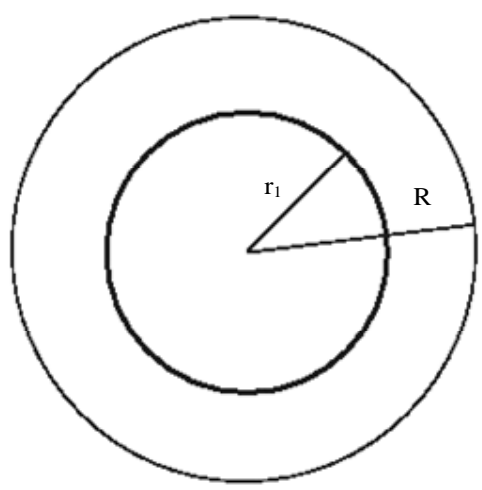

Figure 1. Spherical cloud of gas. 
Solving (4) and (6) with $\phi=0$ when $\rho=0$ we have:

$$
\rho=\phi^{\frac{1}{\gamma-1}} K^{\frac{1}{1-\gamma}}
$$

or

$$
\rho=L \phi^{n}
$$

where $n=\frac{1}{\gamma-1}$ and $L=K^{-n}$. If this value of $\rho$ is replaced into Equation (5), we obtain:

$$
\nabla^{2} \phi=-\delta^{2} \phi^{n}
$$

where, $\delta^{2}=4 \pi L G$.

This is the Lane-Emden equation. This is a second order ordinary differential equation for the density profile $\phi$ as a function of the radius $r_{1}$. We also have to determine initial conditions at $r_{1}=0$. Obviously, at the center the density has to be the central density, and hence $\phi=1$ at $r_{1}=0$. Also, the derivative of the density has to vanish at the center, and therefore $\phi^{\prime}=0$ at $r_{1}=0$.

Our goal is to find a function $\phi$ that satisfies the Lane-Emden Equation (9). In general, this has to be done numerically using the differential transformation method.

The Zhou's Method is a semi-numerical-analytic method for solving ordinary and partial differential equations. The concept of the DTM was first introduced by Zhou in 1986 [2]. Its main application therein is to solve both linear and non-linear initial value problems in electric circuit analysis. This technique constructs an analytical solution in the form of a polynomial. The Taylor series method is computationally expensive for large orders. The differential transformation method is an alternative procedure for obtaining analytic Taylor series solution of the differential equations. The series often coincides with the Taylor expansion of the true solution at point $x_{0}=0$, in the initial value case, although the series can be rapidly converged in a very small region.

Many numerical methods were developed for this type of nonlinear ordinary differential equations, specifically on Lane-Emden type equations such as the Adomian Decomposition Method (ADM) [3,4], the Homotopy Perturbation Method (HPM) [5,6], the Homotopy Analysis Method (HAM) [7] and Bernstein Operational Matrix of Integration [8], in [9] Hojjati and Parand propose a class of second derivative multistep methods (SDMM) for solving some well-known classes of Lane-Emden type equations. Finally in [10] Batiha propose the variational iteration method (VIM) for a class of Lane-Emden type equation, powerful method for the solution of linear and nonlinear equations. In this paper, we show superiority of the DTM by applying them on the some type LaneEmden type equations (white-dwarfs).

\section{Differential Transformation Method}

Differential transformation method of function $y(x)$ is defined as follows:

$$
Y(k)=\frac{1}{k !}\left[\frac{\mathrm{d}^{k} y(x)}{\mathrm{d} x^{k}}\right]_{x=x_{0}}
$$

In (10), $y(x)$ is the original function and $Y(k)$ is the transformed function and the inverse differential transformation is defined as:

$$
y(x)=\sum_{k=0}^{\infty} Y(k) x^{k}
$$

In real applications, function $y(x)$ is expressed by a finite series and Equation (11) can be written as:

$$
y(x)=\sum_{k=0}^{n} Y(k) x^{k}
$$

Equation (12) implies that

$$
\sum_{k=n+1}^{\infty} Y(k) x^{k}
$$

is negligibly small. Here, $n$ is decided by the convergence of natural frequency in this study.

The following theorems that can be deduced from Equations (10) and (11).

Theorem 1.1. If

$$
y(x)=f(x) \pm g(x) \text {, then } Y(k)=F(k) \pm G(k) .
$$

Theorem 1.2. If

$$
y(x)=\alpha_{1} f(x), \text { then } Y(k)=\alpha_{1} F(k),
$$

$\alpha_{1}$ is a constant.

Theorem 1.3. If

$$
y(x)=\frac{\mathrm{d}^{n} g(x)}{\mathrm{d} x^{n}} \text {, then } Y(k)=\frac{(k+n) !}{k !} G(k+n) .
$$

Theorem 1.4. If

$$
y(x)=g(x) h(x) \text {, then } Y(k)=\sum_{k_{1}=0}^{k} G\left(k_{1}\right) H\left(k-k_{1}\right) .
$$

Theorem 1.5. If

$$
y(x)=x^{n}, \text { then } Y(k)=\delta(k-n),
$$

where

$$
\delta(k-n)= \begin{cases}1, & k=n \\ 0, & k \neq n .\end{cases}
$$

Theorem 1.6. (Cárdenas $A$ ). If $y(x)=x^{m} f(x)$ with $m \in \mathbb{N}$, then:

$$
Y(k)= \begin{cases}0, & k<m \\ F(k-m), & k \geq m .\end{cases}
$$

Proof. We proof this theorem by induction method. We assume that is true for $m$, then we will prove that is true also for $m+1$. Using DTM to a product we have:

$$
y(x)=x^{m+1} f(x)=x\left[x^{m} f(x)\right]
$$


then,

$$
Y(k)=\sum_{k_{1}=0}^{k} Y_{1}\left(k_{1}\right) Y_{2}\left(k-k_{1}\right)
$$

where $y_{1}(x)=x$ and $y_{2}(x)=x^{m} f(x)$. Therefore,

$$
Y(k)=\sum_{k_{1}=0}^{k} \delta\left(k_{1}-1\right) Y_{2}\left(k-k_{1}\right)
$$

whenever $k=0$, the expression reduces to:

$$
\delta(-1) Y_{2}(0)=0 .
$$

Now, if $k>0$, then all terms of the sum are zero except $\$ k_{1}=1$ obtaining:

$$
\begin{aligned}
& Y\left[x^{m+1} f(x)\right](k) \\
& =Y\left[x^{m} f(x)\right](k-1)= \begin{cases}0, & k-1<m \\
F(k-1-m), & k-1 \geq m .\end{cases}
\end{aligned}
$$

Rearranging the expression we have:

$$
Y\left[x^{m+1} f(x)\right](k)= \begin{cases}0, & k<m+1 \\ F(k-(m+1)), & k \geq m+1 .\end{cases}
$$

The proofs of Theorems 1-5 are available in [1].

\section{Numerical Solution for $C=0.3$}

To illustrate the ability of the Zhou's method for the Lane-Emden type equation (white-dwarf), the next problem is provided for $C=0.2$. The results reveal that this method is very effective.

Problem. To solve $y^{\prime \prime}+\frac{2}{x} y^{\prime}+\left(y^{2}-C\right)^{\frac{3}{2}}=0$ subject to $y(0)=1$ and $y^{\prime}(0)=0$. First, multiplying both sides by $x$ we have:

$$
x y^{\prime \prime}+2 y^{\prime}+x\left(y^{2}-C\right)^{\frac{3}{2}}=0
$$

Here, is easy to verify that the function

$$
f(y)=\left(y^{2}-C\right)^{\frac{3}{2}}
$$

has a series expansion:

$$
\begin{aligned}
f(y) \approx q^{3}+3 q(y-1)+\frac{3 q^{2}+3}{2 q}(y-1)^{2} \\
+\frac{9 q^{2}-3}{3 ! q^{3}}(y-1)^{3}+\cdots
\end{aligned}
$$

where $q^{2}=1-C$. Therefore, (13) takes the form:

$$
\begin{aligned}
& x y^{\prime \prime}+2 y^{\prime}+x\left[q^{3}+3 q(y-1)+\frac{3 q^{2}+3}{2 q}(y-1)^{2}\right. \\
& \left.+\frac{9 q^{2}-3}{3 ! q^{3}}(y-1)^{3}+\cdots\right]=0
\end{aligned}
$$

Using in (15) the Theorems 3, 4 and 6 we have:

$$
\begin{aligned}
& (k+1) k Y(k+1)+2(k+1) Y(k+1)+\alpha_{1} \delta(k-1) \\
& +\alpha_{2} Y(k-1)+\frac{\alpha_{3}}{2 !} S_{1}+\frac{\alpha_{4}}{3 !} S_{2}+\cdots
\end{aligned}
$$

or

$$
\begin{aligned}
& Y(k+1)=\frac{1}{(k+2)(k+1)} \\
& \cdot\left[-\alpha_{1} \delta(k-1)-\alpha_{2} Y(k-1)-\frac{\alpha_{3}}{2 !} S_{1}-\frac{\alpha_{4}}{3 !} S_{2}-\cdots\right]
\end{aligned}
$$

where,

$$
\begin{aligned}
& \alpha_{1}=q^{3}-3 q+\frac{3 q^{2}+3}{2 q}-\frac{9 q^{2}-3}{3 ! q^{3}}+\cdots \\
& \alpha_{2}=3 q-2 \frac{3 q^{2}+3}{2 q}+3 \frac{9 q^{2}-3}{3 ! q^{3}}+\cdots \\
& \alpha_{3}=\frac{3 q^{2}+3}{2 q}-3 \frac{9 q^{2}-3}{3 ! q^{3}}+\cdots
\end{aligned}
$$

and successively. Also,

$$
\begin{aligned}
& S_{1}=\sum_{k_{1}=0}^{k-1} Y\left(k_{1}\right) Y\left(k-1-k_{1}\right) \\
& \left.S_{2}=\sum_{k_{2}=0}^{k-1} \sum_{k_{1}=0}^{k_{2}} Y\left(k_{1}\right)\right) Y\left(k_{2}-k_{1}\right) Y\left(k-1-k_{2}\right) \\
& S_{3}=\sum_{k_{3}=0}^{k-1} \sum_{k_{2}=0}^{k_{3}} \sum_{k_{1}=0}^{k_{2}} Y\left(k_{1}\right) Y\left(k_{2}-k_{1}\right) \\
& \quad Y\left(k_{3}-k_{2}\right) Y\left(k-1-k_{3}\right)
\end{aligned}
$$

for all $k \geq 1$.

Now, from the initial conditions $y(0)=1$ and $y^{\prime}(0)=0$ we have:

$$
Y(0)=1 \quad \text { and } \quad Y(1)=0
$$

Substituting Equation (21) into Equation (17) and by recursive method, the results are listed as follows:

For $k=1$ we have:

$$
Y(2)=\frac{1}{6}\left[-\alpha_{1}-\alpha_{2}-\frac{\alpha_{3}}{2 !}\right]=-\frac{1}{6} q^{3} .
$$

For $k=2$ we have:

$$
Y(3)=\frac{1}{12}\left[-\alpha_{1} \delta(2-1)-\alpha_{2} Y(1)-\frac{\alpha_{3}}{2 !} S_{1}-\cdots\right]=0
$$

and then, $Y(3)=0$.

For $k=3$ we have:

$$
Y(4)=\frac{1}{20}\left[-\alpha_{1} \delta(3-1)-\alpha_{2} Y(2)-\frac{\alpha_{3}}{2 !} S_{1}-\cdots\right]
$$


and then,

$$
Y(4)=\frac{1}{40} q^{4} .
$$

For $k=4$ we have:

$$
Y(5)=\frac{1}{30}\left[-\alpha_{1} \delta(4-1)-\alpha_{2} Y(3)-\frac{\alpha_{3}}{2 !} S_{1}-\cdots\right]
$$

and then, $Y(5)=0$.

For $k=5$ we have:

$$
Y(6)=\frac{1}{42}\left[-\alpha_{1} \delta(5-1)-\alpha_{2} Y(4)-\frac{\alpha_{3}}{2 !} S_{1}-\cdots\right]
$$

and then,

$$
Y(6)=-\frac{5}{5040} q^{7}-\frac{14}{5040} q^{5} .
$$

Therefore using (12), the closed form of the solution can be easily written as:

$$
\begin{aligned}
y(x) & =\sum_{k=0}^{n} Y(k) x^{k} \\
& =Y(0) x^{0}+Y(1) x+Y(2) x^{2}+Y(3) x^{3} \\
& +Y(4) x^{4}+Y(5) x^{5}+Y(6) x^{6}+\cdots \\
& =1-\frac{1}{6}(1-C)^{\frac{3}{2}} x^{2}+\frac{1}{40}(1-C)^{4} x^{4} \\
& -\left(\frac{5}{5040}(1-C)^{\frac{7}{2}}+\frac{14}{5040}(1-C)^{\frac{5}{2}}\right) x^{6}+\cdots
\end{aligned}
$$

A series solution obtained by Chandrasekhar [2] using series expansion was:

$$
\begin{aligned}
y(x) \cong & 1-\frac{q^{3}}{6} x^{2}+\frac{q^{4}}{40} x^{4}-\frac{q^{5}}{7 !}\left(5 q^{2}+14\right) x^{6} \\
& +\frac{q^{6}}{3 \times 9 !}\left(339 q^{2}+280\right) x^{8}+\cdots
\end{aligned}
$$

Table 1 shows the comparison of $y(x)$ obtained by the DTM (method proposed in this work) and those obtained by Hojjati. The resulting graph of the whitedwarfs equation in comparison to the present method and those obtained by [5] is shown in Figure 2.

\section{Conclusion}

In this work, we presented the definition and handling of one-dimensional differential transformation method. Using the differential transformation, differential equations can be transformed to algebraic equations and the resulting algebraic equations are called iterative equations. This method has applied to solve some class of LaneEmden type equations as a model for the study of the gravitational potential of these degenerate stars (whitedwarfs), which are nonlinear ordinary differential equations on the semi-infinite domain (see [11]). The Figure
Table 1. Comparison between Zhou's method and SDMM.

\begin{tabular}{cccc}
\hline $\boldsymbol{x}$ & $\boldsymbol{D T M}$ & SDMM & Error \\
\hline 0.0 & 1.0000000000 & 1.0000000000 & 0.0000000000 \\
0.5 & 0.9812035800 & 0.912034800 & 0.0000001000 \\
1.0 & 0.9270041568 & 0.9270031568 & 0.0000010000 \\
1.5 & 0.8439248841 & 0.8439247581 & 0.0000001260 \\
2.0 & 0.7425430743 & 0.7425430235 & 0.0000000508 \\
2.5 & 0.6365969111 & 0.6365953025 & 0.0000016086 \\
3.0 & 0.5410635754 & 0.5410633690 & 0.0000002064 \\
3.5 & 0.4691977489 & 0.4691238259 & 0.0000739230 \\
\hline
\end{tabular}

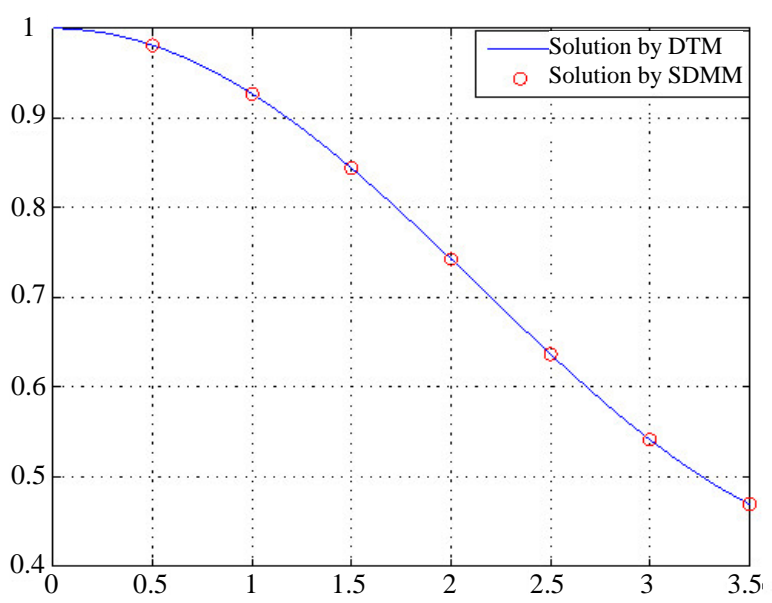

Figure 2. The numerical results $y(x)$ obtained using DTM with $C=0.2$.

2 and table clearly show the high efficiency of DTM to solve nonlinear equations in comparison with other analytical methods equations (see [12,13]).

\section{Acknowledgements}

The authors gratefully acknowledge the support of the Universidad Tecnológica de Pereira and the group GEDNOL and would like to thank the referee for his valuable suggestions that improved the presentation of the paper.

\section{REFERENCES}

[1] S. Chandrasekhar, "Introduction to Study of Stellar Structure,” Dover, New York, 1967.

[2] J. K. Zhou, "Differential Transformation and Its Applications for Electrical Circuits,” Huazhong University of Science \& Technology Press, Wuhan, 1986.

[3] S. J. Liao, "A New Analytic Algorithm of Lane-Emden Type Equations,” Advances Applied Mathematics, Vol. 1, 
No. 142, 2003, pp. 1-16

[4] A. M. Wazwaz, "A New Algorithm for Solving Differential Equations of Lane-Emden Type," Applied Mathematics and Computation, Vol. 118, No. 2-3, 2001, pp. 287310. http://dx.doi.org/10.1016/S0096-3003(99)00223-4

[5] R. A. Gorder, "An Elegant Perturbation Solution for the Lane-Emden Equation of the Second Kind," New Astronomy, Vol. 2, No. 16, 2011, pp. 65-67. http://dx.doi.org/10.1016/j.newast.2010.08.005

[6] J. I. Ramos, "Series Approach to the Lane-Emden Equation and Comparison with the Homotopy Perturbation Method," Chaos Solitons Fractals, Vol. 38, No. 2, 2008, pp. 400-408. http://dx.doi.org/10.1016/j.chaos.2006.11.018

[7] S. Iqbal and A. Javed, "Application of Optimal, Advances,” Applied Mathematics, Vol. 1, No. 42, 2005, pp. 2948.

[8] C. M. Khalique and P. Ntsime, "Exact Solutions of the Lane-Emden Type Equation,” New Astronomy, Vol. 7, No. 13, 2008, pp. 476-480. http://dx.doi.org/10.1016/j.newast.2008.01.002

[9] G. Hojjati and K. Parand, “An Efficient Computational Algorithm for Solving the Nonlinear Lane-Emden Type Equations," International Journal of Mathematics and Computation, Vol. 4, No. 7, 2011, pp. 182-187.

[10] B. Batiha, "Numerical Solution of a Class of Singular Second-Order IVPs by Variational Iteration Method," International Journal of Mathematical Analysis, Vol. 3, No. 40, 2009, pp. 1953-1968.

[11] H. Davis, "Introduction to Nonlinear Differential and Integral Equations,” Dover, New York, 1962.

[12] N. Kumar and R. Pandey, "Solution of the Lane-Emden Equation Using the Bernstein Operational Matrix of Integration,” ISRN Astronomy and Astrophysics, Vol. 2011, 2011, Article ID: 351747. http://dx.doi.org/10.5402/2011/351747

[13] S. Motsa and S. Shate, "New Analytic Solution to the Lane-Emden Equation of Index 2," Mathematics Problems Engineering, Vol. 2012, 2012, Article ID: 614796. 